Check for updates

Cite this: Chem. Sci., 2019, 10, 4077

๑ All publication charges for this article have been paid for by the Royal Society of Chemistry

\section{Direct access to 2-aryl substituted pyrrolinium salts for carbon centre based radicals without pyrrolidine-2-ylidene alias cyclic(alkyl)(amino) carbene (CAAC) as a precursort:}

\author{
Debdeep Mandal, (D) a Sebastian Sobottka, ${ }^{\text {b }}$ Ramapada Dolai, ${ }^{a}$ Avijit Maiti, (D) ${ }^{a}$ \\ Debabrata Dhara, (D) a Pankaj Kalita, (D) C Ramakirushnan Suriya Narayanan, ID a \\ Vadapalli Chandrasekhar, (iD *ad Biprajit Sarkar (iD *b and Anukul Jana (iD *a
}

\begin{abstract}
The synthesis of organic radicals is challenging due to their inherent instability. In recent years, cyclic(alkyl)(amino)carbene (CAAC)-derived 2-substituted pyrrolinium salts have been used as synthons for the synthesis of isolable carbon-based radicals. Herein, we report a direct, easy and convenient method for the synthesis of 2-aryl substituted pyrrolinium salts without using CAAC as a precursor. These cations can be reduced to the corresponding radicals. The influence of the aryl substituent at the $\mathrm{C}-2$ position on radical stabilization and dimerization has been investigated. Because of the large scope of our strategy (capability to modulate different substituents at all the $\mathrm{C}$ - and $\mathrm{N}$-centres of the pyrrolinium salts), it has the merit to be an extremely effective and productive route for generating carbon-based radicals whose stability as well as reactivity can be varied.
\end{abstract}

Received 7th December 2018

Accepted 27th February 2019

DOI: $10.1039 / \mathrm{c} 8 \mathrm{sc} 05477 \mathrm{k}$

rsc.li/chemical-science

\section{Introduction}

Radicals play crucial roles in various chemical and biological processes, ${ }^{1}$ as well as in industrial processes such as in polymer chemistry. ${ }^{2}$ Recent reports have also suggested their extensive use as catalysts and reactive intermediates to synthesize various types of molecules. ${ }^{3}$ However, because of their inherent instability and high reactivity most radicals exist as very short lived species. To understand the nature and reactivity of radicals formed in various reactions as intermediates their stabilization and isolation are very crucial. Efforts in this regard include trapping unstable radicals by coupling with other stable radicals ${ }^{4}$ or quenching them with suitable reagents. ${ }^{5}$ In recent years, cyclic(alkyl)(amino) carbenes (CAACs) have been used for the isolation of different kinds of radicals including those that are carbon based

${ }^{a}$ Tata Institute of Fundamental Research Hyderabad, Gopanpally, Hyderabad-500107, Telangana, India.E-mail: ajana@tifrh.res.in

${ }^{b}$ Institut für Chemie und Biochemie, Anorganische Chemie, Freie Universität Berlin, Fabeckstraße 34-36, 14195, Berlin, Germany. E-mail: biprajit.sarkar@fu-berlin.de

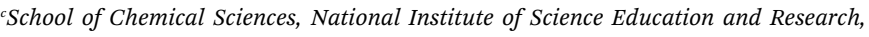
HBNI, Bhubaneswar-752050, India

${ }^{d}$ Department of Chemistry, Indian Institute of Technology Kanpur, Kanpur-208016, India. E-mail: vc@iitk.ac.in

$\dagger$ Dedicated to Professor Vinod K. Singh on the occasion of his 60th birthday. \$ Electronic supplementary information (ESI) available: Experimental details, NMR spectra, X-ray crystallographic data and theoretical details. CCDC 1868053 $\left(3^{\mathrm{H}}\right), 1868056\left(3^{\mathrm{Me}}\right), 1868057\left(3^{\mathrm{Et}}\right), 1868055\left(3^{\mathrm{tBu}}\right)$ and $1868054\left(3^{\mathrm{H}_{2}}\right)$. For ESI and crystallographic data in CIF or other electronic format see DOI: $10.1039 / \mathrm{c} 8 \mathrm{sc} 05477 \mathrm{k}$
(Scheme 1), ${ }^{6}$ as well as hetero-atom based systems. ${ }^{7}$ In particular, Bertrand $e t$ al. have recently reported the syntheses of CAACderived mixed valence (radical and cation) organic compounds. ${ }^{8}$

So far all the reported CAAC-scaffold-based carbon radicals such as IV are generated from their corresponding pyrrolidinium salts, $\mathbf{V}$, derived from CAAC, VI (Scheme 2). However, a serious limitation and disadvantage of using CAACs as precursors for generating radicals is their very limited number, ${ }^{9}$ which severely restricts the variation of the substituent scope in the products. To get CAAC-scaffold-based carbon centre radicals, the synthesis of 2-substituted pyrrolinium salts as immediate precursors is crucial (Scheme 2). In other words, if the synthesis of 2-substituted pyrrolinium salts can be achieved through alternate ${ }^{10}$ and more convenient routes, without using CAAC, this will enrich the library of 2-substituted pyrrolinium salts as well as the corresponding radicals. Therefore, a new methodology for synthesizing 2-substituted pyrrolinium salts, $\mathbf{V}$, with a wide substrate scope is urgently required.

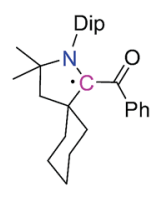

I

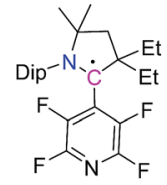

II

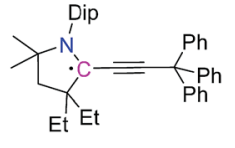

III
Scheme 1 Selected chemical structures of CAAC-derived $\alpha$-carbonyl radical I, ${ }^{6 a}$ tetrafluoropyridyl radical II, ${ }^{6 c}$ and propargyl radical III. ${ }^{6 f}$ 


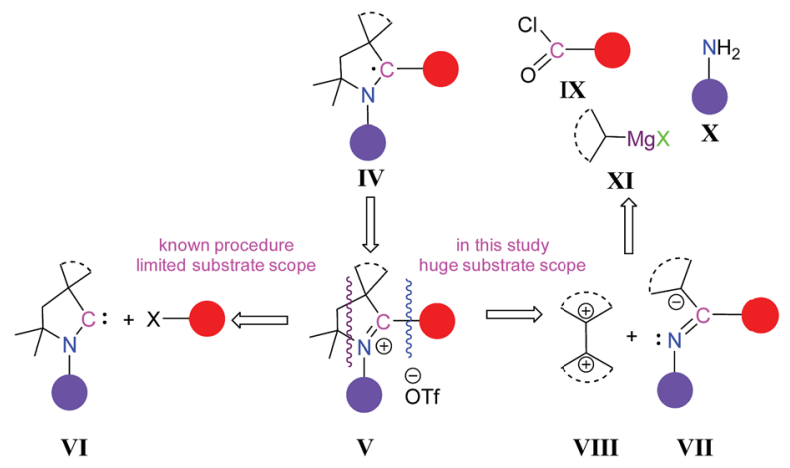

Scheme 2 Reported strategy and our strategy for the synthesis of 2aryl substituted pyrrolinium slats, $\mathrm{V}$.

\section{Results and discussion}

Herein, we have considered a new strategy where 2-substituted pyrrolinium salts, $\mathbf{V}$, can be generated from a secondary ketimine, VII, which has only one $\alpha$-hydrogen, and a compound having geminal electrophilic centres, VIII. The secondary ketimine, VII, can be obtained in three very convenient steps (Scheme 2), from commercially available reagents: acid chloride, IX; primary amine, $\mathbf{X}$; and secondary Grignard reagents, $\mathrm{R}_{2} \mathrm{CHMgX}, \mathrm{XI}^{11}$ In this strategy, there is a huge scope for variation at all the $C$-centres as well as at the $N$-centre of the pyrrolinium ring. Moreover, the expansion of the five membered ring is also possible.

To test our strategy, we considered 4-alkylbenzoyl chlorides, $\mathbf{1}^{\mathbf{R}}$, which are commercially available, as the starting precursors, keeping in mind that the 4-alkylphenyl substituent will be at the C-2 position of pyrrolinium salts which can influence the stability as well as the self-association of the radicals, which may be generated. Moreover, this choice will give access to the first non-fluorinated 2-aryl-substituted pyrrolinium salts and the subsequent radicals derived from them. For $N$-substitution we have considered the $i$ Pr-group.

The reaction of $\mathbf{1}^{\mathbf{R}}$ with $i \mathrm{PrNH}_{2} / \mathrm{Et}_{3} \mathrm{~N}, \mathrm{PCl}_{5}$, and $i \mathrm{PrMgCl}$ sequentially leads to the imine, $2^{\mathbf{R}}$, as a colorless liquid (Scheme 2). ${ }^{12}$ Subsequently, the reaction of $2^{\mathbf{R}}$ with $n \mathrm{BuLi}$, isobutylene oxide and triflic anhydride sequentially in one pot leads to the formation of 2 -aryl substituted pyrrolinium triflates, $3^{\mathbf{R}}$, as white solids (Scheme 3). ${ }^{12}$

All the 2 -aryl substituted pyrrolinium triflates, $3^{\mathbf{R}}$, were characterized by NMR spectroscopy as well as by single-crystal X-ray measurements (see Fig. 1 for the molecular structure of $3^{\mathbf{H}}$ and Fig. S1-S3 for the molecular structures of $3^{\mathrm{Me}}, 3^{\mathrm{Et}}$, and $3^{t \mathrm{Bu}}$ in the ESI:). An examination of the molecular structures

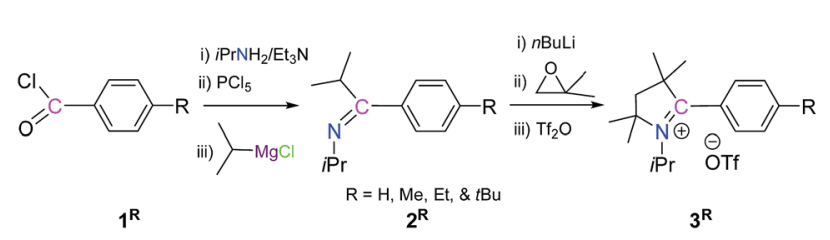

Scheme 3 Synthesis of 2-aryl substituted pyrrolinium triflate, $3^{R}$.

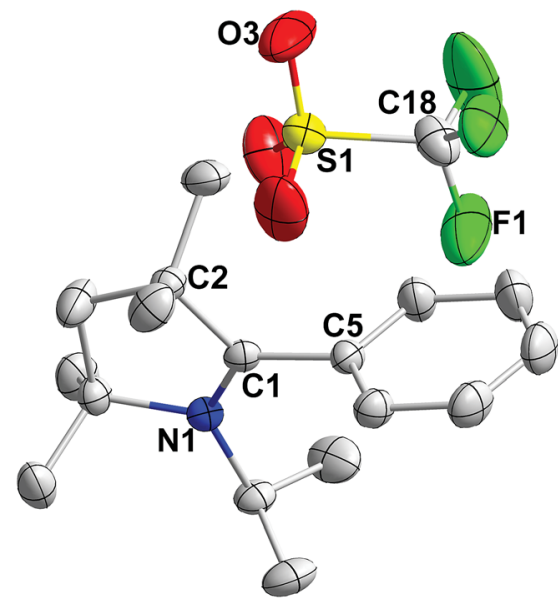

Fig. 1 Solid state molecular structure of $3^{\mathrm{H}}$, with thermal ellipsoids at $30 \%$. All hydrogens are omitted for clarity. Selected bond lengths $(\AA)$ and angles $\left({ }^{\circ}\right)$ : N1-C1 1.275(3), C1-C2 1.508(3), C1-C5 1.486(3), and N1-C1-C2 113.34(19).

reveals that the pyrrolinium- and the phenyl rings are almost perpendicular to each other (torsion angle: $86.93^{\circ}$ for $3^{\mathbf{H}}, 89.60^{\circ}$ for $3^{\mathrm{Me}}, 87.55^{\circ}$ for $3^{\mathrm{Et}}$, and $90^{\circ}$ for $\left.3^{t \mathrm{Bu}}\right) .{ }^{12}$

The cyclovoltammograms of 2-aryl substituted pyrrolinium triflates, $3^{\mathbf{R}}$, reveal two reduction steps, the first event at around $E_{1 / 2}=-1.8 \mathrm{~V}\left(v s . \mathrm{FcH} / \mathrm{FcH}^{+}\right)$which is completely reversible on the cyclic voltammetry timescale and indicates the formation of a neutral radicals, $3^{\mathbf{R}^{\cdot}}$ (Fig. 2). The substituents on the phenyl rings do not have any significant influence on the redox potentials of these compounds. The second reduction step is irreversible and is seen around $E_{1 / 2}=-2.53$ to $-2.75 \mathrm{~V}$ indicating the formation of a 2-e reduced anionic compounds, $3^{\mathbf{R}-}$, which could not be generated chemically (Fig. 2) ${ }^{6 c}$ Furthermore, the second reduction step has a profound influence on the reversibility of the first reduction step even on the

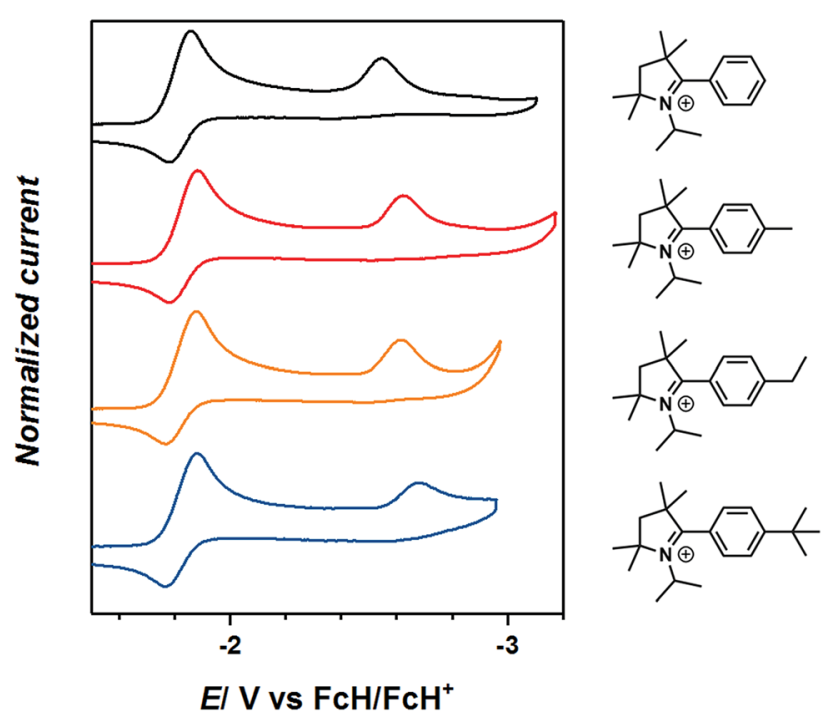

Fig. $2 \mathrm{CV}$ of $3^{\mathrm{R}}$ in $\mathrm{THF} / 0.1 \mathrm{NBu}_{4} \mathrm{PF}_{6}$ measured at a $\mathrm{GC}$ working electrode at $100 \mathrm{mV} \mathrm{s}^{-1}$ 
timescale of the cyclic voltammetry experiments (see Fig. S17S21 in the ESI $\$$ ).

In order to chemically access the species that are generated electrochemically, we carried out the reduction of $\mathbf{3}^{\mathbf{H}}$ with $\mathrm{KC}_{8}$ in THF (Scheme 4), which leads to a dark red solution from which pale yellow crystals could be harvested after keeping the $n$-hexane extraction at $-35{ }^{\circ} \mathrm{C}$, overnight. Analysis through a single crystal X-ray diffraction study showed the formation of a dimer, $3^{\mathbf{H}_{2}}$ (Fig. 3), which must have been formed by a head-totail dimerization of the initially formed radical, $3^{\mathbf{H}^{*}}$ as in the case of the Gomberg triphenylmethyl radical, $\mathrm{Ph}_{3} \mathrm{C}^{\cdot} .^{13}$ The formation of the radical, $3^{\mathbf{H}^{*}}$ in solution was confirmed by ESR, which reveals a signal centered at $g=2.003$ (Fig. 4). Compound, $3^{\mathbf{H}_{2}}$ exists in equilibrium, in solution, with the monomer, $3^{\mathbf{H}^{\mathbf{}}}$. Also there is a possibility of formation of $3^{\mathbf{H}_{2}}$ by a coupling between the starting cation, $3^{\mathbf{H}}$, and the 2 -electron reduced compound, $3^{\mathbf{H}-}$, which has been observed in a spectroelectrochemistry study. ${ }^{\mathbf{1 2}}$ This is analogous to the reactivity of the trityl cation which forms adducts with neutral bases. ${ }^{14}$ Both mechanisms were considered for simulating the cyclic voltammograms and this provided the best fits with the experimental voltammograms (see Fig. S33-S37 in the ESIt).

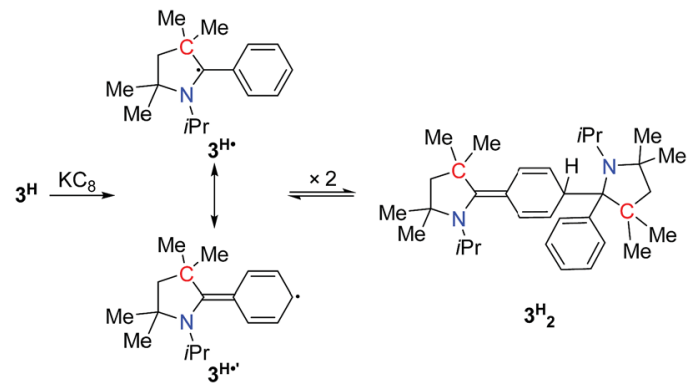

Scheme 4 Synthesis of $3^{\mathrm{H}_{2}}$.

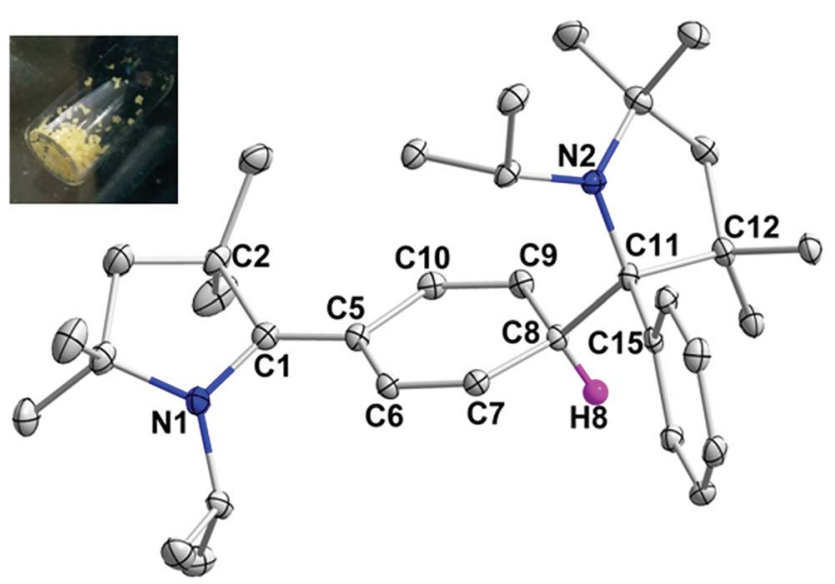

Fig. 3 Molecular structure of $3^{\mathrm{H}_{2}}$, with thermal ellipsoids at $30 \%$ and all hydrogens except $\mathrm{C} 8-\mathrm{H}$ are omitted for clarity. Selected bond lengths (Å) and angles ( ${ }^{\circ}$ ): C1-C5 1.370(4), C5-C10 1.450(5), C10-C9 1.331(4), C9-C8 1.513(4), C8-C11 1.603(4), and C11-C15 1.545(4); N1-C1-C2 108.4(3), C5-C1-N1 124.2(3), C5-C1-C2 127.2(3), C9-C8-C11 114.8(2), C7-C8-C11 114.9(2), and N2-C11-C12 102.9(2) (inset: yellow crystalline compounds of $3^{\mathrm{H}_{2}}$ ).
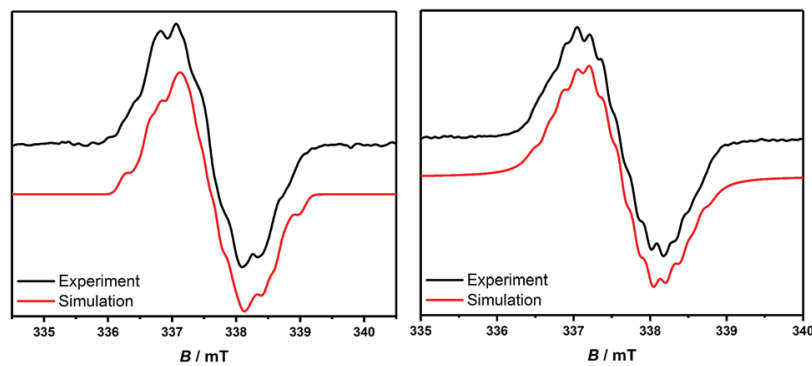

Fig. 4 EPR spectra of $3^{\mathrm{H}^{*}}$ and $3^{t \mathrm{Bu}}{ }^{\circ}$ in $0.1 \mathrm{M} \mathrm{NBu}_{4} \mathrm{PF}_{6}$ in THF.

Apart from the head-to-tail dimerization of $3^{\mathbf{H}^{*}}$, there is a possibility of tail-to-tail and head-to-head coupling, the latter being very unlikely because of steric reasons. Theoretical calculations reveal that head-to-tail dimerization is the energetically favourable process. On the other hand, the formation of the tail-to-tail dimer is an endothermic process and is destabilized compared to the head-to-tail dimer (see Table S15 and Fig. S52 in the ESI ).

Subsequently, we carried out the reduction of other 2-aryl substituted pyrrolinium salts with $\mathrm{KC}_{8}$ (Scheme 5), where the substitution at the 4-position of the aryl group can block the dimerization. ${ }^{15}$ Both $3^{\mathbf{M e}}$ and $3^{\text {Et }}$ afford a mixture of decomposition products at room temperature although the reduction of $3^{\mathbf{E t}}$ immediately results in a red solution at room temperature. In the case of $3^{t \mathbf{B u}}, \mathrm{KC}_{8}$ reduction produced a deep red color solution at about $-50{ }^{\circ} \mathrm{C}$ which remains sufficiently intense until its work up at room temperature. Thus, after removing the THF from the reaction mixture, we extracted the residue with dry $n$-hexane. Keeping this extract at $-80{ }^{\circ} \mathrm{C}$ afforded deep red crystals (Scheme 5). Unfortunately, these crystals were polycrystalline in nature. Several attempts to grow single crystals in solvents of various polarities like THF and toluene failed to give suitable single crystals for X-ray diffraction study.

Theoretical calculations reveal $3^{\mathbf{M e}^{*}}, 3^{\mathbf{E t}}$, and $3^{\mathbf{t B u}^{*}}$ to be more stable than their corresponding higher energy dimers. ${ }^{12}$ This is the reason why $3^{\mathbf{M e}}$ and $3^{\mathbf{E t}^{*}}$ afford decomposed products rather than the dimer. Due to the steric bulk $3^{\text {tBu}}$ is relatively more stable. The formation of $3^{\mathbf{t} \mathbf{B u}^{\cdot}}$ in situ in solution has been confirmed by ESR spectroscopy by the observation of a signal at $g=2.003$ similar to what was seen for $\mathbf{3}^{\mathbf{H}^{*}}$ (Fig. 4). The hyperfine couplings to the relevant nuclei are likely not resolved due to unfavorable hyperfine coupling/line-width ratios. However, for $3^{t \mathbf{B u}}$ a partial resolution of the hyperfine couplings is observed in the experimental spectrum. The calculated spin densities at the PBE0/IGLO-III level of theory reveal a localization of the spin densities at the C-2 and the $\mathrm{N}-1$ atoms of the CAAC-scaffold with
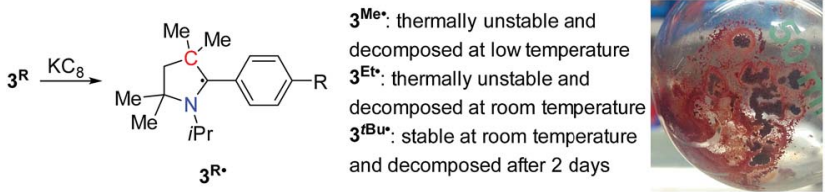

Scheme 5 Reduction of $3^{\mathrm{Me}}, 3^{\mathrm{Et}}$, and $3^{\mathrm{tBu}}$ (left) and picture of $3^{\mathrm{tBu}}$ (right). 

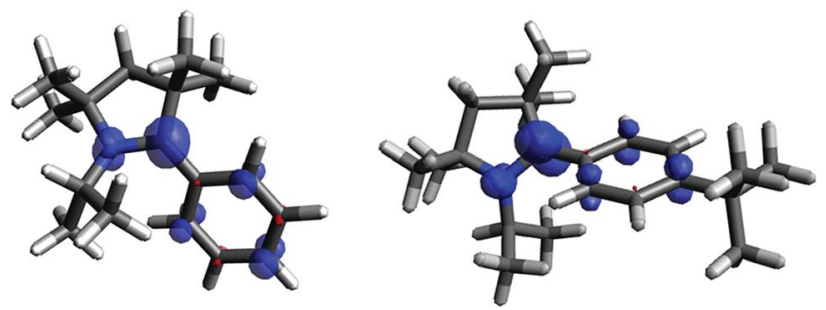

Fig. 5 Spin densities of $3^{\mathrm{H}^{*}}$ (left) and $3^{\text {tBu }}$ (right) at PBEO/IGLO-III, isovalue 0.01

only minor contributions at the phenyl substituent at the C2 position (Fig. 5).

In order to gain insight into the redox properties and the electronic structures of the various redox states (spectro)electrochemical and (TD-)DFT calculations were performed on the 2-pyrrolinium salts, $3^{\mathbf{R}}$. In the UV/vis spectra all the four 2-pyrrolinium salts are transparent in the visible region, as expected for such colorless compounds. However, the in situ generated one-electron-reduced neutral forms (measured exemplarily for two compounds) $3^{\mathbf{H}^{\cdot}}$ and $3^{t \mathbf{B u}^{*}}$ each display one absorption at around $350 \mathrm{~nm}$ and a second broad absorption at around $485 \mathrm{~nm}$ (see Fig. S58, S59 and Table S19 in the ESI:). The UV/vis spectroelectrochemical measurements clearly display the reversible nature of the first reduction step, as seen by the regeneration of the starting spectrum with quantitative intensities on reversing the potential back to the starting potential (see Fig. S57-S60 in the ESIt). The first reduction step is thus also seen to be reversible on the spectroelectrochemical time scale. A full TD-DFT calculation using B3LYP reproduces the bands of both the cationic and the neutral radical forms with reasonable accuracy (see Fig. S66 in the ESI:).

On performing a second reduction on the compounds via in situ UV/vis spectroelectrochemistry the bands at around 350 and $485 \mathrm{~nm}$ that belong to the radical form disappear, and the spectra become more or less transparent in the visible region except for a weak shoulder at 330 or $380 \mathrm{~nm}$ (see Table S19 in the ESI:). As discussed above for the cyclic voltammetry measurements, this second reduction step is not reversible, and switching the potential back to the starting potential does not lead to the regeneration of the starting spectra. Furthermore, on scanning the potential back, the spectra corresponding to the one-electron reduced compounds are not regenerated either. ESR spectroelectrochemistry at the second reduction potential shows a disappearance of the signal corresponding to the radical species, possibly indicating the generation of a diamagnetic compound. These measurements thus point to a drastic change in the structure of the compounds on reducing them with two electrons.

\section{Conclusions}

In conclusion, we have disclosed a new strategy for the synthesis of $1(N)$ - and 2-substituted pyrrolinium salts which are immediate precursors for the synthesis of CAAC-scaffold based carbon centre radicals. This strategy allows a large variation of substrate scope at all the $C$ - and $N$-centres of the pyrrolinium moieties. As a proof of principle, we demonstrated the synthesis of 1(N)-isopropyl-2-(4-R-phenyl)-pyrrolinium triflates $(\mathrm{R}=\mathrm{H}$, $\mathrm{Me}$, Et, and $t \mathrm{Bu})$. These compounds can be converted to the corresponding neutral radicals which were characterized by electrochemical, in situ UV/vis, and spectroelectrochemical (ESR) methods as well as with DFT calculations. In the case of the 2-phenyl substituted radical, the formation of a head-to-tail dimer was observed as in the case of the Gomberg triphenylmethyl radical. In view of the above results, we believe that our strategy will be useful for the synthesis of different CAACscaffold based carbon centre radicals. Currently, we are actively pursuing this strategy to realize new carbon-centered multi-radical centres including diradicaloids.

\section{Conflicts of interest}

There are no conflicts to declare.

\section{Acknowledgements}

This work is supported by the TIFR Hyderabad. V. C. is thankful to the DST for a National J. C. Bose fellowship. The highperformance computing facilities at ZEDAT, FU-Berlin, are acknowledged for access to computing resources.

\section{Notes and references}

1 (a) T. L. Dormandy, Ann. R. Coll. Surg. Engl., 1980, 62, 188194; (b) B. Halliwell and J. M. C. Gutteridge, Free Radicals in Biology and Medicine, Oxford University Press, 2015; (c) M. Hayyan, M. A. Hashim and I. M. AlNashef, Chem. Rev., 2016, 116, 3029-3085.

2 P. Nesvadba, Encyclopedia of Radicals in Chemistry, Biology and Materials, ed. C. Chatgilialoglu and A. Studer, Wiley, 2012, vol. 4, pp. 1701-1736.

3 (a) C. P. Jasperse, D. P. Curran and T. L. Fevig, Chem. Rev., 1991, 91, 1237-1286; (b) M. Yan, J. C. Lo, J. T. Edwards and P. S. Baran, J. Am. Chem. Soc., 2016, 138, 12692-12714.

4 (a) D. H. R. Barton, V. N. L. Gloahec and J. Smith, Tetrahedron Lett., 1998, 39, 7483-7486; (b) E. G. Janzen, Acc. Chem. Res., 1971, 4, 31-40.

5 I. H. Leave and G. C. Ramsay, Tetrahedron, 1969, 25, 56695675.

6 Selected references are: (a) J. K. Mahoney, D. Martin, C. E. Moore, A. L. Rheingold and G. Bertrand, J. Am. Chem. Soc., 2013, 135, 18766-18769; (b) K. C. Mondal, H. W. Roesky, M. C. Schwarzer, G. Frenking, I. Tkach, H. Wolf, D. Kratzert, R. Herbst-Irmer, B. Niepötter and D. Stalke, Angew. Chem., Int. Ed., 2013, 52, 1801-1805; (c) J. K. Mahoney, D. Martin, F. Thomas, C. E. Moore, A. L. Rheingold and G. Bertrand, J. Am. Chem. Soc., 2015, 137, 7519-7525; (d) S. Styra, M. Melaimi, C. E. Moore, A. L. Rheingold, T. Augenstein, F. Breher and G. Bertrand, Chem.-Eur. J., 2015, 21, 8441-8446; (e) D. Munz, J. Chu, M. Melaimi and G. Bertrand, Angew. Chem., Int. Ed., 2016, 55, 12886-12890; $(f)$ D. Mandal, R. Dolai, N. Chrysochos, 
P. Kalita, R. Kumar, D. Dhara, A. Maiti, R. S. Narayanan, G. Rajaraman, C. Schulzke, V. Chandrasekhar and A. Jana, Org. Lett., 2017, 19, 5605-5608; $(g)$ M. M. Hansmann, M. Melaimi and G. Bertrand, J. Am. Chem. Soc., 2017, 139, 15620-15623.

7 Selected references are: (a) P. Bissinger, H. Braunschweig, A. Damme, I. Krummenacher, A. K. Phukan, K. Radacki and S. Sugawara, Angew. Chem., Int. Ed., 2014, 53, 73607363; (b) B. Li, S. Kundu, C. Stückl, H. Zhu, H. Keil, R. Herbst-Irmer, D. Stalke, B. Schwederski, W. Kaim, D. M. Andrada, G. Frenking and H. W. Roesky, Angew. Chem., Int. Ed., 2017, 56, 397-400; (c) R. Kinjo, B. Donnadieu, M. A. Celik, G. Frenking and G. Bertrand, Science, 2011, 333, 610-613; (d) O. Back, M. Ali Celik, G. Frenking, M. Melaimi, B. Donnadieu and G. Bertrand, $J$. Am. Chem. Soc., 2010, 132, 10262-10263.

8 M. M. Hansmann, M. Melaimi and G. Bertrand, J. Am. Chem. Soc., 2018, 140, 2206-2213.

9 M. Melaimi, R. Jazzar, M. Soleilhavoup and G. Bertrand, Angew. Chem., Int. Ed., 2017, 56, 10046-10068.

10 U. S. D. Paul and U. Radius, Chem.-Eur. J., 2017, 23, 39934009.
11 H. G. Richey Jr, K. Narasaka, M. Yamane, Y. Chen and W. Tang, Encyclopedia of Reagents for Organic Synthesis, 2017. 12 See the ESIt for the details.

13 (a) M. Gomberg, J. Am. Chem. Soc., 1900, 22, 757-771; (b) H. Lankamp, W. T. Nauta and C. MacLean, Tetrahedron Lett., 1968, 9, 249-254; (c) C. Lichtenberg, L. Viciu, M. Vogt, R. E. Rodríguez-Lugo, M. Adelhardt, J. Sutter, M. M. Khusniyarov, K. Meyer, B. de Bruin, E. Billd and H. Grützmacher, Chem. Commun., 2015, 51, 13890-13893; (d) S. Rösel, J. Becker, W. D. Allen and P. R. Schreiner, J. Am. Chem. Soc., 2018, 140, 14421-14432; (e) S. Rösel, C. Balestrieri and P. R. Schreiner, Chem. Sci., 2017, 8, 405410.

14 (a) P. A. Chase, A. L. Gille, T. M. Gilbert and D. W. Stephan, Dalton Trans., 2009, 7179-7188; (b) L. Cabrera, G. C. Welch, J. D. Masuda, P. Wei and D. W. Stephan, Inorg. Chim. Acta, 2006, 359, 3066-3071; (c) E. Follet, P. Mayer, D. S. Stephenson, A. R. Ofial and G. Berionni, Chem.-Eur. J., 2017, 23, 7422-7427.

15 (a) T. H. Colie and E. S. Lewis, J. Am. Chem. Soc., 1979, 101, 1810-1814; (b) D. Dünnebacke, W. P. Neumann, A. Penenory and U. Stewen, Chem. Ber., 1989, 122, 533-535. 